\title{
RESEARCH
}

Open Access

\section{Comparing the simultaneous determination of cis- and trans-palmitoleic acid in fish oil using HPLC and GC}

Huang Wen-wen ${ }^{1,2^{* \dagger}} \mathbb{D}$, Hong Bi-hong ${ }^{2 \dagger}$, Sun Ji-peng ${ }^{2}$, Tan Ran ${ }^{2}$, Bai Kai-kai ${ }^{2}$, Yang Ting $^{3}$, Wu Hao ${ }^{1 *}$ and Yi Rui-zao $2^{*}$

\begin{abstract}
Background: Cis- and trans-palmitoleic acids (Cis-POA and trans-POA) are isomers of palmitoleic acid, a monounsaturated fatty acid which affects glucose and lipid metabolism, and reduces insulin resistance. Trans-POA is used as a biomarker for indicating the risk of type II diabetes and coronary heart disease, but no methods of analysis or distinguishing between cis-POA and trans-POA have yet been reported.

Method: An accurate and precise HPLC method was developed to determine cis- and trans-POA simultaneously, and compared with results from a GC method. Cis- and trans-POA were analyzed by HPLC on a reverse-phase BDSC18 column, equilibrated and eluted with acetonitrile (A) and water (B). In the established and validated GC method used for comparison, potassium hydroxide ester exchange was chosen to derivatize the cis- and trans-POA, before being determined.

Results: The calibration curves for cis- and trans-POA were linear over the range 0.05 to $500 \mu \mathrm{g} / \mathrm{mL}$. The HPLC method exhibited good sensitivity, precision and accuracy. The limits of detection (LOD) for cis- and trans-POA were 0.2 and $0.05 \mu \mathrm{g} / \mathrm{mL}$, respectively. The method successfully determined cis- and trans-POA in fish oil. For the GC method, the contents of cis-POA quantified were similar to those from the HPLC method, but the contents of trans-POA revealed significant variation between the two methods.

Conclusions: After a comprehensive consideration of the characteristics of the saponification and methyl esterification methods which have been tested and verified, the HPLC method was found to be suitable for determining cis- and trans-POA contents in fish oil. It was also suggested that in natural fish oil, cis-POA may be in the glyceride state, and trans-POA almost completely in the free acid form. In comparison with the GC method, the HPLC method provided a simpler process and faster analyses for identifying and determining cis- and trans-POA. The study has also provided technical support for studying the pharmacological differences and relationship between structure and activity of cis- and trans-POA. This could help physicians to analyze patients' samples more quickly in $10 \mathrm{~min}$ and therefore provide a more rapid diagnosis of problems relating to the risk of type II diabetes and coronary heart disease.
\end{abstract}

Keywords: Cis-palmitoleic acid, Trans-palmitoleic acid, HPLC, GC, Fish oil

\footnotetext{
*Correspondence: wwhuang@tio.org.cn; whao5795@vip.sina.com; yiruizao@163.com

tWenwen Huang and Bihong Hong contributed equally to this work.

'College of Pharmacy, Nanjing University of Chinese Medicines, Nanjing

210023, People's Republic of China

${ }^{2}$ Third Institute of Oceanography, Ministry of Natural Resources, Xiamen

361005, People's Republic of China

Full list of author information is available at the end of the article
}

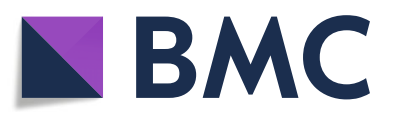

(c) The Author(s). 2019 Open Access This article is distributed under the terms of the Creative Commons Attribution 4.0 International License (http://creativecommons.org/licenses/by/4.0/), which permits unrestricted use, distribution, and reproduction in any medium, provided you give appropriate credit to the original author(s) and the source, provide a link to the Creative Commons license, and indicate if changes were made. The Creative Commons Public Domain Dedication waiver (http://creativecommons.org/publicdomain/zero/1.0/) applies to the data made available in this article, unless otherwise stated. 


\section{Highlights}

- A rapid HPLC method was developed to identify and determine cis- and trans-POA simultaneously in $10 \mathrm{~min}$

- The results for cis-POA quantified in fish oil were similar from both the HPLC and GC methods

- The contents of trans-POA revealed significant variation between the HPLC and GC methods

- Cis-POA may be in the glyceride state, with transPOA almost completely in the free acid form in natural fish oil

\section{Background}

Palmitoleic acid (C16:1, n-7, POA) is a natural omega-7 monounsaturated fatty acid, which is abundant in plant and fish oils [1]. Its two isomers, cis-POA and trans-POA have different spacial structures (Fig. 1). Cis-POA is common in POA from natural sources, and has been demonstrated to favorably influence glucose and lipid metabolism through various mechanisms $[2,3]$. Cis-POA affects the key enzymes during blood glucose metabolism, regulates insulin secretion in humans, and reduces insulin resistance $[4,5]$. Orally-administered cis-POA has been reported to induce satiety, enhance the release of satiety hormones, and decrease the food intake in mice [6]. In mice, cis-POA also reduced body weight gain, ameliorated the development of hyperglycemia and hypertriglyceridemia, and improved insulin sensitivity [7]. However, trans-fatty acids are the subject of ongoing discussion regarding their positive and negative associations with metabolic and cardiovascular risk factors [8]. A higher proportion of trans-POA in plasma phospholipids has been shown to improve insulin sensitivity and decrease the onset of type II diabetes disease [9]. Trans-POA, which also regulates glycolipid metabolism, has been used as a biomarker for indicating the risk of type II diabetes and coronary heart disease, with a correlation between trans-POA and low-density lipoprotein being reported [10-12]. Generally, cis- and trans-POA

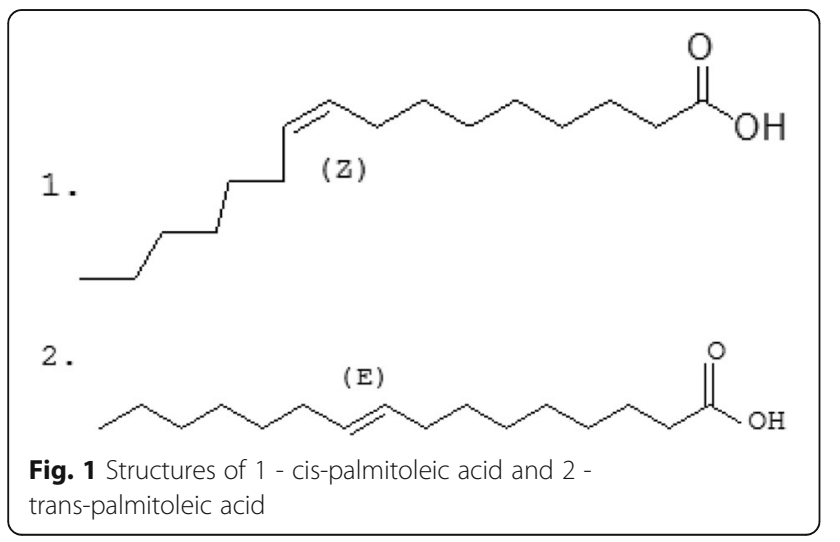

both have therapeutic effects in chronic diseases such as diabetes, metabolic syndrome and inflammation with different mechanisms of activity and both are rich in fish oil. However, how the mechanisms of the physiological activity of cis- and trans-POA differ is not clear.

Fatty acids are usually analyzed using GC or GC-MS. The content of cis-POA was determined as $47.8 \%$ of the total fatty acids of the fruits of sea buckthorn (Hippophae rhamnoides L.) [13], and $6.4 \%$ of the total fatty acids of Bupleurum Chinense DC from China determined by GC-MS and GC-FID [14]. Usually, fatty acids, such as DHA and EPA from fish oil or other sources have been analyzed by GC or GC-MS [15-17]. Cis-palmitoleic acids can be analyzed simultaneously by common methods such as GC and GC-MS, but no method has been reported for identifying and determining cis- and trans-POA using HPLC or HPLC-MS simultaneously. Thus, developing a method for the quantitative analyses of cis- and transPOA simultaneously is important for future pharmacological studies and applications.

Overall, this study aims to report the development, validation, and application of a rapid and sensitive method for the detection and quantitation of cis- and trans-POA in fish oil using HPLC and GC methods. Comparing these methods will reveal which method will be more suitable for determining cis- and trans-POA simultaneously in fish oil. The method developed will need to be sensitive and selective, with a wide range of detection and low limit of detection.

\section{Materials and methods Materials and reagents}

Standard samples of cis-palmitoleic acid and trans-palmitoleic acid were purchased from Nu-Chek-Prep Inc. (Elysian, MN, USA), and HPLC-grade acetonitrile, n-hexane and methanol from Merck KGaA (Darmstadt, Germany). Ultrapure water was produced by a Millipore Milli-Q system (Millipore Corp., Billerica, MA, USA). The mixed crude fish oil was purchased from Xiamen Kingdomway Group Co. (Xiamen, Fujian province, China) and fresh mackerel were purchased locally in Xiamen.

\section{Extraction and preparation of fish oil samples}

A 100-g portion of mackerel flesh was weighed and crushed then placed in a conical flask with $1 \mathrm{~L}$ dichloromethane. After stirring for ten $\mathrm{h}$, the supernatant dichloromethane was transferred to a round flask. This procedure was repeated three times. The respective supernatants were combined, then concentrated by removing the solvent to obtain the crude fish oil.

"Using a method of saponification modified from published procedures [13-18], a 2-g sample of crude fish oil was weighed and added to $3 \mathrm{~g}$ potassium hydroxide, 
$24 \mathrm{~mL}$ methanol solution and $20 \mathrm{~mL}$ water, then stirred and heated for $2 \mathrm{~h}$ at $80^{\circ} \mathrm{C}$. After adjusting the $\mathrm{pH}$ to 3 with sulfuric acid, the mixture was centrifuged with $5000 \mathrm{~g}$ so that the mixed free fatty acids could be removed and concentrated by removing the solvent.

A sample of mixed free fatty acids was weighed (0.05 g), $1 \mathrm{~mL}$ methanol solution was added then passed through a Millex $0.2-\mu \mathrm{m}$ nylon membrane syringe filter before analysis.

\section{Liquid chromatography conditions}

The analytes were separated on an Agilent UPLC-1290 system (Agilent Corp., Milford, MA, USA) using a DBS C18 column $(100 \times 4.6 \mathrm{~mm}, 2.4 \mu \mathrm{m}$, Thermo Fisher Scientific, Lafayette, CO, USA). The mobile phase was composed of acetonitrile (A) and water $(B),(A: B=80: 20$, $v / \mathrm{v})$ at a flow rate of $1 \mathrm{~mL} / \mathrm{min}$ and the injection volume was $10 \mu \mathrm{L}$. The detection wavelength was set at $205 \mathrm{~nm}$.

\section{Stock solutions and working solutions}

Individual standard stock solutions of cis- and trans-POA (1.112 and $1.457 \mathrm{mg} / \mathrm{mL}$, respectively) were prepared in acetonitrile. These stock solutions were mixed then serially diluted with acetonitrile to provide standard working solutions at six different concentrations of cis- and trans-POA from 0.05 to $500 \mu \mathrm{g} / \mathrm{mL}$. All solutions were stored at $-20^{\circ} \mathrm{C}$ then brought to room temperature before use.

\section{Method validation}

The method was validated for linearity, limits of quantification and detection (LOQs and LODs), precision, repeatability, recovery and stability following the International Conference on Harmonization guidelines [19]. A series of standard solutions was prepared to determine the linearity of the analytes. Linearity was evaluated by using six calibration points in the concentration range from 0 to $500 \mu \mathrm{g} / \mathrm{mL}$. The LOQ for each analyte, cis- and trans-POA, was determined at a signalto-noise $(\mathrm{S} / \mathrm{N})$ ratio of about 3 and 10 , respectively. Precision was evaluated by six injections of the standard solution within one day. Intra- and inter-day variations were chosen to determine the repeatability of the method. The mixed standard solutions were analyzed using six replicates within $24 \mathrm{~h}$ for intra-day variation, and for the inter-day variability test, the solutions were determined in triplicate for three consecutive days. Recovery was evaluated by spiking the samples by adding mixed standard samples at low $(0.5 \mu \mathrm{g} / \mathrm{mL})$, medium $(5 \mu \mathrm{g} / \mathrm{mL})$, and high $(50 \mu \mathrm{g} / \mathrm{mL})$ levels (three replicates at each concentration level) to fish oil samples, which had previously been analyzed. All variation was expressed in terms of relative standard deviation (RSD).
The average recovery was estimated using the following formula:

Recovery $(\%)=[($ detection quantity-original quantity $) /$ quantity added] $\times 100$.

\section{Determination of cis- and trans-POA by GC method}

Using a modified potassium hydroxide ester exchange method [20-23], a 0.1-g sample of crude fish oil was weighed then added to $1 \mathrm{~mL}$ of $1 \mathrm{M}$ potassium hydroxide-methanol solution (without water) and $4 \mathrm{~mL}$ n-hexane, heated for $30 \mathrm{~min}$ at $40^{\circ} \mathrm{C}$. The supernatant solution was passed through a Millex 0.2- $\mu \mathrm{m}$ nylon membrane syringe filter before injection.

The contents of cis- and trans-POA were determined using a validated GC method recently successfully established in our laboratory (Fig. 3).

\section{Statistical analysis}

All experiments were performed at least three times. Analyses of variance (ANOVA) were performed using the Student-Newman-Keuls (S-N-K) procedure to test the significance of differences between mean values using IBM SPSS Statistics software version 23.0 (IBM Corp., Armonk, NY, USA).

\section{Results}

\section{Optimization of HPLC conditions}

The chromatographic conditions, in particular the analytical column, column temperature, the composition of the mobile phase, and injection volume, were optimized through several trials to obtain chromatograms with a satisfactory resolution, appropriate retention times, and high sensitivity. The BDS C18 column was selected as it produced satisfactory separations and peak shapes with good resolution. Various mobile phases, such as methanol or acetonitrile and water, at different solvent ratios (60:40, 70:30, 80:20, 90:10), were tested for their sensitivity and good chromatographic behavior. The results showed that using a mobile phase of acetonitrile and water (80:20) was most efficient and produced symmetrical peaks for both analytes. The peak times and run times for determining cis- and trans-POA using the HPLC method of 4.6-5.1 min, and $10 \mathrm{~min}$, respectively, (Fig. 2) were much shorter than those using the GC method of 48.3-49.1 min, and $90 \mathrm{~min}$, respectively (Fig. 3).

\section{Validation of the analysis method}

Standard curves were established by plotting the ratios of the chromatogram peak areas for cis- and trans-POA. These curves exhibited correlation coefficients greater than 0.999 and good linearity over concentration ranges from 0.5 to $500 \mu \mathrm{g} / \mathrm{mL}$ for cis- and trans-POA. Typical calibration equations were:

$$
y=3012 x-831.27(r=0.9991) \text { for cis-POA, and. }
$$



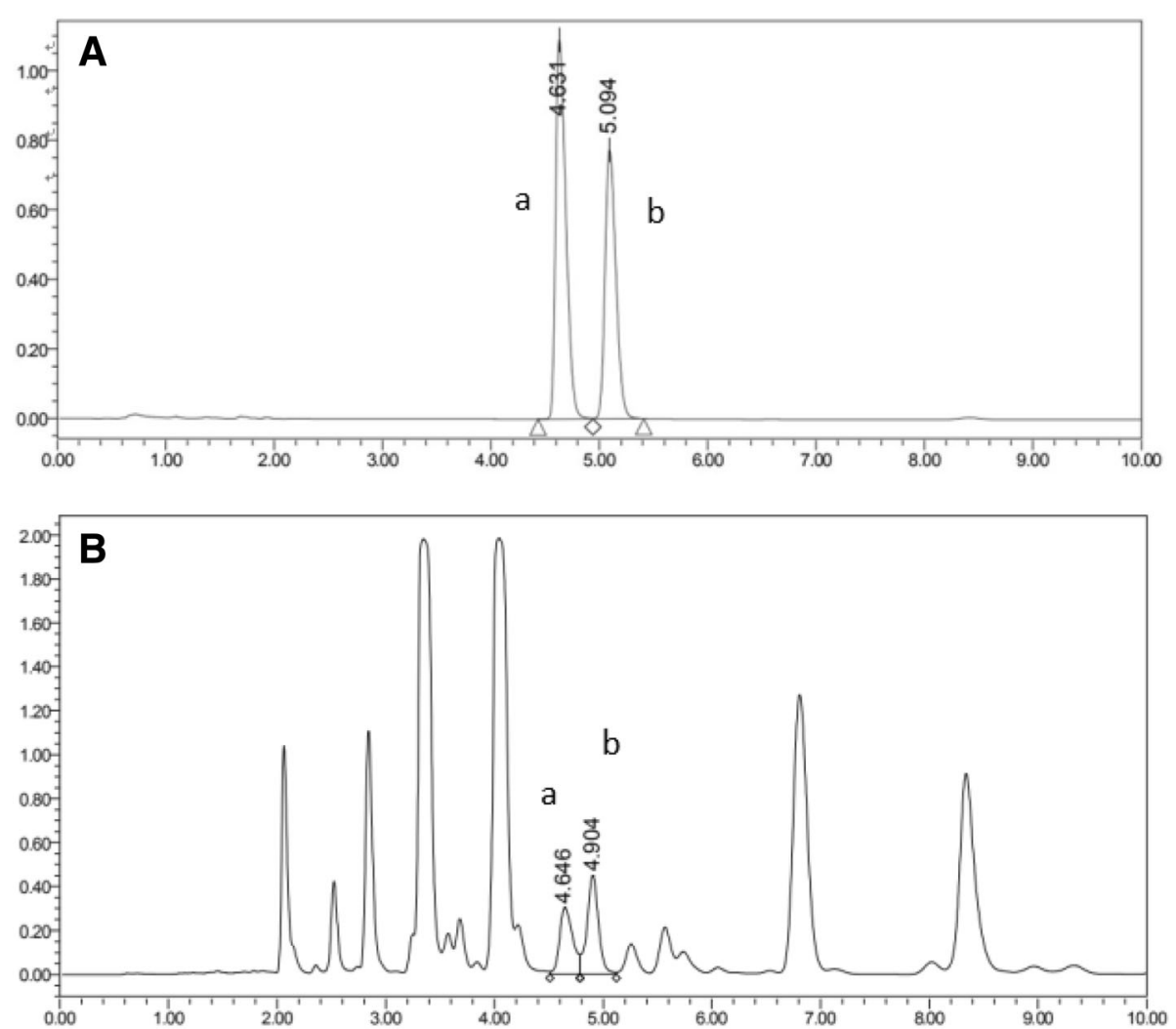

Fig. 2 HPLC chromatogram of (A) cis- and trans-POA standard samples and (B) fish oil sample. a: cis-palmitoleic acid, b: trans-palmitoleic acid

$y=3949.6 x+58.628(r=0.9999)$ for trans-POA, where $y$ represents the peak area ratio of an analyte, and $\mathrm{x}$ represents an analyte concentration.

The lower limits of quantification (LLOQ) for cis- and trans-POA were defined as 0.5 and $0.2 \mu \mathrm{g} / \mathrm{mL}$, respectively, based on a signal-to-noise ratio $(\mathrm{S} / \mathrm{N})$ of 10 . The limit of detection (LOD) was estimated to be 0.2 and $0.05 \mu \mathrm{g} / \mathrm{mL}$, respectively, based on an $\mathrm{S} / \mathrm{N}$ of 3 .

The results for precision, repeatability, stability and recovery of cis and trans-POA are summarized in Tables 1 and 2 . The mean relative standard deviation (RSD) values for intra-day and inter-day variation were 1.26 and $0.96 \%$ for cis-POA, and 1.10 and $0.63 \%$ for trans-POA, respectively. The HPLC method also had an acceptable accuracy with an average recovery of 94.12 and $103.61 \%$ for cis- and trans-POA, respectively. This demonstrated that the HPLC method developed was sufficiently reliable and accurate to be suitable for quantifying cis- and trans-POA in fish oil.

\section{Sample analysis}

The HPLC and GC methods were successfully applied to the quantification of cis- and trans-POA in fish oil, and the results are summarized in Table 3 . The results reveal significant variation in the contents of the cisand trans-POA quantified in fish oil by both the HPLC and GC methods. In particular, the contents of trans-POA in the mackerel and mixed fish oils were 6.49, and 6.73\%, respectively, using HPLC and 0.41, and $0.21 \%$, respectively, using GC. Such variation may have been caused by incomplete methyl esterification. After repeated verification and validation using standard samples, it was found that triglyceride-POA, resulting from the potassium hydroxide ester exchange method, allowed easy methylation but when cis- and trans-POA were in the free acid state they could hardly be methylated.

\section{Discussion}

HPLC and GC methods have been used extensively for half a century for lipid analysis [20-22]. Although GC has been used most widely for analyzing and determining fatty acids, the application of HPLC has increased recently with both methods having characteristic strengths and weaknesses. Usually fatty acid samples have been detected and quantified by the GC method. To reduce the residue of fatty acids in the chromatographic column caused by burning at high temperature, the fatty acids have been almost completely methyl esterified, a crucial process for achieving high levels of accuracy and precision of determination. 

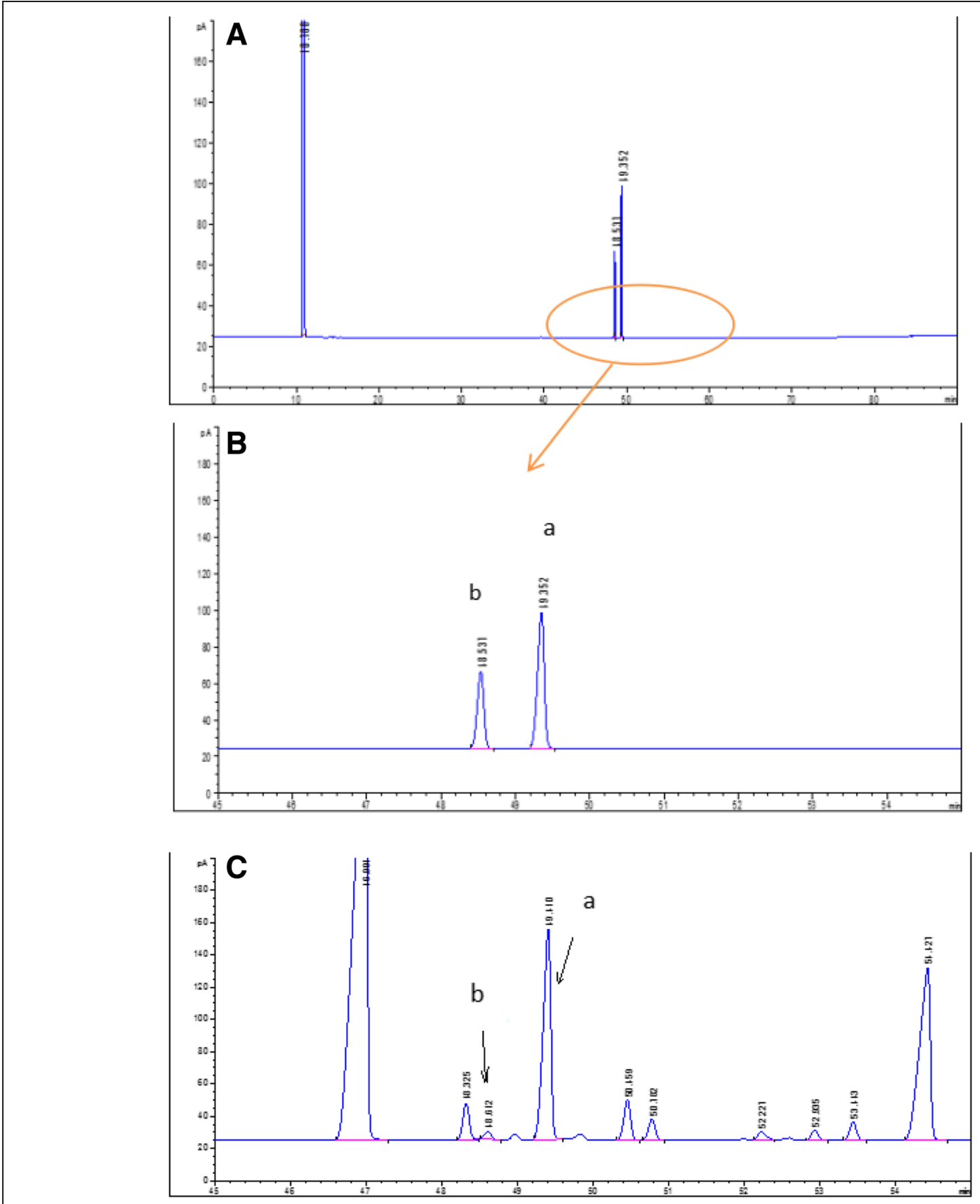

Fig. 3 HPLC chromatogram of A, B: cis- and trans-POA standard samples and C: fish oil sample. a: cis-palmitoleic acid, b: trans-palmitoleic acid

Table 1 Precision, repeatability and stability for determination of cis and trans-POA contents of fish oil using HPLC

\begin{tabular}{|c|c|c|c|c|c|c|}
\hline \multirow[t]{2}{*}{ Analyte } & \multirow{2}{*}{$\begin{array}{l}\text { Precision } \\
\text { (RSD, \%, } \\
n=6)\end{array}$} & \multicolumn{4}{|c|}{$\begin{array}{l}\text { Repeatability } \\
(\mathrm{RSD}, \%, n=6)\end{array}$} & \multirow{2}{*}{$\begin{array}{l}\text { Stability } \\
\text { (RSD, \%, } \\
n=6)\end{array}$} \\
\hline & & Day 1 & Day 2 & Day 3 & Inter-day & \\
\hline Cis-POA & 1.48 & 1.26 & 0.89 & 1.25 & 0.96 & 1.05 \\
\hline Trans-POA & 0.98 & 0.57 & 0.63 & 1.10 & 0.63 & 0.79 \\
\hline
\end{tabular}

Current methods for methyl esterification include potassium hydroxide ester exchange $[20,21]$, the use of hydrochloric acid [22, 24], or boron trifluoride [25-27] and the methyl sulfate method [23]. Free fatty acids in the glyceride state are hardly methyl esterified using the potassium hydroxide ester exchange method but fatty acids in the glyceride state are easily methyl esterified. However, the boron trifluoride methyl esterification method has been reported as insufficient for derivatizing 
Table 2 Recovery of cis and trans-POA for determination of cis and trans-POA contents of fish oil using HPLC

\begin{tabular}{lcccc}
\hline Analyte & Low & Medium & High & Average \\
& Recovery \pm RSD (\%) & Recovery \pm RSD (\%) & Recovery \pm RSD (\%) & Recovery \pm RSD (\%) \\
\hline Cis-POA & $93.37 \pm 3.12$ & $94.77 \pm 3.31$ & $94.62 \pm 1.43$ & $94.12 \pm 3.26$ \\
Trans-POA & $98.72 \pm 2.92$ & $105.91 \pm 2.63$ & $106.96 \pm 1.61$ & $103.61 \pm 2.76$ \\
\hline
\end{tabular}

both free fatty acids and fatty acids in the glyceride state [23]. In contrast, methanolic hydrochloric acid $(\mathrm{HCl})$ as well as a combination of $\mathrm{BF}_{3}$ with methanolic sodium hydroxide $\left(\mathrm{NaOH}+\mathrm{BF}_{3}\right)$ has been reported as suitable for the derivatization of free fatty acids, polar lipids, and triglycerides (derivatization rate $>80 \%$ for all tested lipids) [23].

In the present study, the potassium hydroxide ester exchange method was chosen to derivatize the cis- and trans-POA. It was found that the results from the HPLC and GC methods were different, because the free fatty acids were not derivatized in the presence of potassium hydroxide, a result agreeing with Ostermann et al. [23]. It was suggested that cis-POA may be in the glyceride state, while the trans-POA was almost completely present as a free fatty acid in natural fish oil, so that the derivatization rate using any method of methyl esterification with cis- and trans-POA in fish oil would be different. Therefore, the HPLC method was considered to be more suitable for analyzing cis- and trans-POA in the free fatty acids state simultaneously by saponification. Thus, the HPLC method would be simpler and more accurate than $\mathrm{GC}$, because it does not require the derivatization step.

The presence of cis- and trans-POA in natural fish oil in different states or forms is related to the differences in their biological activity. This study provides technical support for the study of these pharmacological differences and the relationship between the structure and activity of cis- and trans-POA. With its lower limits of quantification and wider range of quantification, the HPLC method has been shown to be suitable for detecting many types of compound, such as palmitoleic acid-methyl ester, palmitoleic acid-ethyl ester, and palmitoleic acid-glyceride, simultaneously. This method could also be used to analyze cis- and trans-POA in plasma or other biological tissue, to help physicians analyze patients' samples quickly, in $10 \mathrm{~min}$, and therefore

Table 3 The content of cis- and trans-POA in crude fish oil

\begin{tabular}{|c|c|c|c|c|}
\hline & \multicolumn{4}{|c|}{ Content (\%) in crude fish oil } \\
\hline & \multicolumn{2}{|c|}{ Extracted from Mackerel fish } & \multicolumn{2}{|c|}{ Purchased (Mixed fish oil } \\
\hline & Cis-POA & Trans-POA & Cis-POA & Trans-POA \\
\hline HPLC method & 7.93 & 6.49 & 5.26 & 6.73 \\
\hline GC method & 7.54 & 0.41 & 4.26 & 0.21 \\
\hline
\end{tabular}

provide a more rapid diagnosis of problems relating to the risk of type II diabetes and coronary heart disease.

\section{Conclusions}

A highly sensitive, reproducible and accurate HPLC method has been developed for comprehensively determining cis- and trans-POA contents in fish oil. The contents of the quantified cis-POA were similar using the HPLC and GC methods, but those of trans-POA were significantly different. A comprehensive consideration of the characteristics of saponification and methyl esterification suggested that the HPLC method was more suitable for determining cis- and trans-POA contents in fish oil, and that in natural fish oil, cis-POA may be in the glyceride state while trans-POA was a free fatty acid.

\section{Abbreviations}

Cis-POA: Cis-palmitoleic acid; GC: Gas chromatography; HPLC: High-pressure liquid chromatography; POA: Palmitoleic acid; Trans-POA: Trans-palmitoleic acid

\section{Acknowledgements}

Thanks to Professor Wu Hao for her warm guidance and support. The assistance of the Department of Pharmacology, School of Pharmacy, Nanjing University of Chinese Medicine, and the Third Institute of Oceanography, Ministry of Natural Resources, P.R. China are all gratefully acknowledged. We thank Philip Creed, PhD, from Liwen Bianji, Edanz Group China (www.

liwenbianji.cn/ac), for editing the English text of a draft of this manuscript.

\section{Funding}

This work was supported by the Scientific Research Foundation of the Third Institute of Oceanography, State Oceanic Administration, China [grant number 2016040], Agricultural guidance projects of Fujian Province, China [grant number 2017 N0017], Science and Technology Plan Program of Fujian Province, China [grant number 2017Y0101], the National Natural Science Fund, China [grant number 81870138].

\section{Availability of data and materials}

The data sets used and/or analyzed during the current study are available from the corresponding author on reasonable request.

\section{Authors' contributions}

Wenwen Huang and Bihong Hong carried out the experiments and prepared the manuscript. Jipeng Sun, Ran Tan, Kaikai Bai, Ting Yang, Ruizao Yi and Hao Wu contributed with valuable discussions and scientific input. Wenwen Huang conducted the experimental design. All authors helped to prepare the manuscript and approved the final version.

\section{Authors' information}

a. College of Pharmacy, Nanjing University of Chinese Medicines, Nanjing 210023, PR China, b. Third Institute of Oceanography, Ministry of Natural Resources, Xiamen 361,005, PR China, c. Department of Hematology, Fujian Medical University Union Hospital, Fujian Provincial Key Laboratory on Hematology, Fujian Institute of Hematology, Fuzhou 350,001, PR China. 
Ethics approval and consent to participate

Not applicable.

\section{Consent for publication}

Not applicable.

\section{Competing interests}

The authors declare no conflict of interest.

\section{Publisher's Note}

Springer Nature remains neutral with regard to jurisdictional claims in published maps and institutional affiliations.

\section{Author details}

${ }^{1}$ College of Pharmacy, Nanjing University of Chinese Medicines, Nanjing 210023, People's Republic of China. ${ }^{2}$ Third Institute of Oceanography, Ministry of Natural Resources, Xiamen 361005, People's Republic of China. ${ }^{3}$ Department of Hematology, Fujian Medical University Union Hospital, Fujian Provincial Key Laboratory on Hematology, Fujian Institute of Hematology, Fuzhou 350001, People's Republic of China.

Received: 23 January 2019 Accepted: 26 March 2019

Published online: 04 April 2019

\section{References}

1. Morgan NG, Dhayal S. Unsaturated fatty acids as cytoprotective agents in the pancreatic beta-cell. Prostaglandins Leukot Essent Fatty Acids. 2010;82:231-6.

2. Matthan NR, Dillard A, Lecker $J L, I p$ B, Lichtenstein AH. Effects of dietary palmitoleic acid on plasma lipoprotein profile and aortic cholesterol accumulation are similar to those of other unsaturated fatty acids in the F1B golden Syrian hamster. J Nutr. 2009;139:215-21.

3. Misra A, Singhal N, Khurana L. Obesity, the metabolic syndrome, and type 2 diabetes in developing countries: role of dietary fats and oils. J Am Coll Nutr. 2010;29:289S-301S.

4. Souza CO, Teixeira AAS, Lima EA. Palmitoleic acid (n-7) attenuates the immunometabolic disturbances caused by a high-fat diet independently of PPARalpha. Mediat Inflamm. 2014:582197-7.

5. Bergman BC, Howard D, Schauer IE. The importance of palmitoleic acid to adipocyte insulin resistance and whole-body insulin sensitivity in type I diabetes. J Clin Endocr Metab. 2013;98(1):E40-50.

6. Yang ZH, Takeo J, Katayama M. Oral administration of omega-7 palmitoleic acid induces satiety and the release of appetite-related hormones in male rats. Appetite. 2013:1-7.

7. Yang $\mathrm{ZH}$, Miyahara $\mathrm{H}$, Hatanaka A. Chronic administration of palmitoleic acid reduces insulin resistance and hepatic lipid accumulation in KK-ay mice with genetic type 2 diabetes. Lipids Health Dis. 2011;10:120.

8. Brouwer IA, Wanders AJ, Katan MB. Trans fatty acids and cardiovascular health: research completed. Eur J Clin Nutr. 2013;67:541-7.

9. Jaudszus A, Kramer R. Trans palmitoleic acid arises endogenously from dietary vaccenic acid. Am J Clin Nutr. 2014;99:431-5.

10. Mozaffarian D, Otto d OOMC, Lemaitre RN, Fretts AM, Hotamisligil G, Tsai MY, Siscovick DS, Nettleton JA. Trans-Palmitoleic acid, other dairy fat biomarkers, and incident diabetes: the multi-ethnic study of atherosclerosis (MESA). Am J Clin Nutr. 2013;97(4):854-61.

11. Mozaffarian D, Cao HM, King IB. Trans-Palmitoleic acid, metabolic risk factors, and new-onset diabetes in US adults. Ann Intern Med. 2010; 153(12):790-9.

12. Nunes EA, Rafacho A. Implications of palmitoleic acid (palmitoleate) on glucose homeostasis, insulin resistance and diabetes. Curr Drug Targets. 2017;18:619-28.

13. Cakir A. Essential oil and fatty acid composition of the fruits of Hippophae rhamnoides L. (sea buckthorn) and Myrtus communis L. from Turkey. Biochem Syst Ecol. 2004;32:809-16.

14. Li XQ, Song AH, Li W. Analysis of the fatty acid from Bupleurum Chinense DC in China by GC-MS and GC-FID. Chem Pharmaceut Bull. 2005;53(12):1613-7.

15. Yi T, Li SM, Fan JY. Comparative analysis of EPA and DHA in fish oil nutritional capsules by GC-MS. Lipids Health Dis. 2014;13(1):190.

16. Wang QF, Liu T. Determination of EPA and DHA in cod-liver oil by GC. Chin J Mod Appl Pharm. 2018;35(9).
17. Peng FJ, Yang WH. Determination of DHA and linoleic acid contents in infant formula Milk powder by GC-MS. Food Sci. 2011;32(14):261-3.

18. Haug A, Olesen I, Christophersen OA. Individual variation and intraclass correlation in arachidonic acid and eicosapentaenoic acid in chicken muscle. Lipids Health Dis. 2010;9(9):37.

19. Branch SK. Guidelines from the international conference on harmonisation (ICH). J Pharmaceut Biomed. 2005;38(5):798-805.

20. Sanders $T A B$, Younger KM. The effect of dietary-supplements of Omega-3 poly-unsaturated fatty-acids on the fatty-acid composition of platelets and plasma choline Phosphoglycerides. Brit J Nutr. 1981;45:613-6.

21. Hammond EW. Chromatographic techniques for lipid analysis. Trends Anal Chem. 1989;8:308-17.

22. Shantha NC, Napolitano GE. Gas chromatography of fatty acids. J Chromatogr. 1992;624:37-51.

23. Ostermann A, Müller M, Willenberg I, Schebb NH. Determining the fatty acid composition in plasma and tissues as fatty acid methyl esters using gas chromatography - a comparison of different derivatization and extraction procedures. Prostag Leukotr Ess. 2014;91(6):235-41.

24. Li S, Li L, Cao J. Improvement of pre-column derivatization for the determination of eicosapentaenoic acid and docosahexaenoic acid in fish oil soft capsules by gas chromatography. Food Safe Qual Detec Technol. 2016;7(3):951-7.

25. Cai WJ, Zhang XJ, Su ZL. Determination of eicosapentaenoic acid, docosahexaenoic acid, and docosapentaenoic acid in fish oil by gas chromatography. Food Safe Qual Detec Technol. 2015;6(5):1924-8.

26. 3023. GB 5009. 168-2016, Determination of Fatty acids in Foods of the National Standard for Food Safety. 2017.

27. Kratz M, Cullen P, Kannenberg F, Kassner A, Fobker M, Abuja PM, Assmann $G$, Wahrburg U. Effects of dietary fatty acids on the composition and oxidizability of low-density lipoprotein. Eur J Clin Nutr. 2002;56:72-81.

Ready to submit your research? Choose BMC and benefit from:

- fast, convenient online submission

- thorough peer review by experienced researchers in your field

- rapid publication on acceptance

- support for research data, including large and complex data types

- gold Open Access which fosters wider collaboration and increased citations

- maximum visibility for your research: over $100 \mathrm{M}$ website views per year

At BMC, research is always in progress.

Learn more biomedcentral.com/submissions 